\title{
Internação e mortalidade hospitalar de vítimas de queimaduras no Brasil
}

\section{Hospitalization and hospital mortality of burn victims in Brazil}

\author{
William Campo Meschial' • Dirléia Florentino dos Santos ${ }^{2} \bullet$ Aroldo Gavioli $^{3} \bullet$ Vivian Carla de Castro ${ }^{4}$ \\ Muriel Fernanda de Lima $^{5} \bullet$ Magda Lúcia Félix de Oliveira ${ }^{6}$
}

\begin{abstract}
RESUMO
Objetivo: analisar a evolução das internações e da taxa de mortalidade hospitalar de vítimas de queimaduras no Brasil, no período 2007-2016. Método: estudo observacional retrospectivo, com dados do Sistema de Informações Hospitalares do Sistema Único de Saúde. Empregou-se o teste qui-quadrado, sendo estimado o risco relativo de óbito. Resultados: Houve maior risco de óbito por queimaduras decorrentes da exposição à fumaça, ao fogo e às chamas (RR 2,3; IC95\% 2,25; 2,4I), na região Sudeste (RR I,4; IC95\% I,36; I,44) e em idosos com 80 anos e mais (RR 4,9; IC95\% 4,74;5,08). Predominaram as internações e mortalidade hospitalar decorrentes da exposição à corrente elétrica, à radiação e às temperaturas e pressões extremas do ambiente (84, I\% e 78,8\%), nas regiões sul e sudeste (50,4\%), na faixa-etária de 20 a 59 anos (58,2\%) e no sexo masculino (68,9\%). Conclusões: houve aumento das internações e taxa de mortalidade hospitalar por queimaduras com o avançar dos anos, sendo observadas importantes diferenças sociodemográficas e geográficas, revelando importantes particularidades dos acidentes por queimaduras. Destaca-se a importância de exaltar a casuística de maneira mais aprofundada, uma vez que tal conhecimento é primordial para discussão de políticas de saúde de cunho preventivo e assistencial.

Palavras-chave: Queimaduras; Hospitalização; Mortalidade Hospitalar; Saúde Pública; Causas Externas; Epidemiologia.
\end{abstract}

\begin{abstract}
Aim: to analyze the evolution of hospitalizations and the hospital mortality rate of burn victims in Brazil, in the period 20072016. Method: retrospective observational study, using data from the Hospital Information System of the Unified Health System. The chi-square test was used, estimating the relative risk of death. Results: There was a higher risk of death from burns resulting from exposure to smoke, fire and flames ( $R R$ 2.3; 95\% Cl 2.25; $2.4 \mathrm{I})$, in the Southeast ( $\mathrm{RR} \mathrm{I.4;95 \%} \mathrm{Cl} \mathrm{I,} \mathrm{36;} \mathrm{I.44)}$ and in the elderly aged 80 and over (RR 4.9; $95 \% \mathrm{Cl} 4.74 ; 5.08)$. Hospitalizations and hospital mortality due to exposure to electric current, radiation and the extreme temperatures and pressures of the environment ( $84.1 \%$ and $78.8 \%)$, in the south and southeast regions (50.4\%), in the 20 to 59 years old (58.2\%) and male (68.9\%). Conclusions: there was an increase in hospital admissions and a hospital mortality rate due to burns over the years, with important sociodemographic and geographical differences, revealing important particularities of burn accidents. The importance of knowing this series in more depth is emphasized, since such knowledge is essential for the discussion of health policies of a preventive and assistance nature. Keywords: Burns; Hospitalization; Hospital Mortality; Public Health; External Causes; Epidemiology.
\end{abstract}

I Enfermeiro. Doutor em Enfermagem. Docente do curso de Graduação em Enfermagem da Universidade do Estado de Santa Catarina. Chapecó, Santa Catarina, Brasil.

2 Enfermeira. Mestra em Enfermagem. Docente do curso de Graduação em Enfermagem da Faculdade Santa Maria da Glória. Maringá, Paraná, Brasil.

3 Enfermeiro. Doutorando em Enfermagem pela Universidade Estadual de Maringá. Hospital Universitário Regional de Maringá. Maringá, Paraná, Brasil.

4 Enfermeira. Doutora em Enfermagem. Docente do curso de Graduação em Medicina do Centro Universitário Integrado, Campo Mourão, Paraná, Brasil.

5 Enfermeira. Doutora em Enfermagem. Docente do curso de Graduação em Enfermagem da Universidade Estadual de Maringá. Maringá, Paraná, Brasil.

6 Enfermeira. Doutora em Saúde Coletiva. Docente do curso de Graduação em Enfermagem e do Programa de Pós-Graduação em Enfermagem da Universidade Estadual de Maringá. Maringá, Paraná, Brasil.

Autor correspondente:William Campo Meschial - Endereço: Rua Sete de Setembro, 77D - Centro, Chapecó, Santa Catarina, Brasil. CEP: 89806-I52. E-mail: williameschial@yahoo.com.br 


\section{INTRODUÇÃO}

Lesões por queimaduras constituem um dos agravos de maior negligência dentre causas externas no Brasil. Em contrapartida, nos países desenvolvidos, observa-se a redução nas taxas de morbimortalidade, decorrente de medidas preventivas e avanços alcançados no manejo ao paciente queimado(').

Em relação à casuística, dados apontam que a incidência de vítimas de queimaduras submetidas à atenção à saúde em todo o mundo alcança aproximadamente II milhões de pessoas, com mais de $\mathbf{3 0 0}$ mil mortes por ano(2). Segundo a Organização Mundial da Saúde, acidentes com fogo estão entre as principais causas de morte entre crianças e adultos jovens, que compreendem idades entre cinco e 29 anos $^{(3)}$.

Apesar da alta morbidade por queimaduras no Brasil e de uma população superior a 207 milhões de habitantes, o país possui ínfimas 56 unidades de tratamento de queimaduras (UTQ), distribuídas de maneira iníqua em 18 dos seus 26 estados e no Distrito Federal. Cabe destacar que a região Norte do país, composta por sete estados e uma população superior a 17 milhões de habitantes não possui nenhuma dessas unidades. Além disso muitas UTQs atuam com recursos humanos e materiais limitados ${ }^{(4)}$.

No Brasil, com o propósito de conhecer o impacto e concentrar o máximo de informações dos dados de morbidade e mortalidade, foram instituídos o Sistema de Informações Hospitalares do SUS (SIHSUS) e o Sistema de Informação sobre Mortalidade (SIM). A centralização dos dados de morbimortalidade tornou possível a monitoração de situações de saúde da população, além de fornecer subsídios para o planejamento das mesmas. No entanto, queimaduras não constituem um agravo de notificação compulsória no país. Logo, nos atendimentos que não resultam em hospitalização, não há a obrigatoriedade de notificação do evento.

As lesões por queimaduras constituem um sério problema de saúde pública em países em desenvolvimento, como o Brasil. Nesse sentido, é relevante o conhecimento das características epidemiológicas dessas injúrias. $O$ levantamento e divulgação dos resultados encontrados neste estudo podem fornecer subsídios para elaboração de programas de prevenção e tratamento de lesões, e constituem um mecanismo para a comparação entre casuísticas nacionais e internacionais ${ }^{(5)}$.

A ampliação do conhecimento acerca da magnitude das queimaduras na população brasileira é necessária, considerando a lacuna de estudos sobre a temática, especialmente dados epidemiológicos, sendo que a maioria das pesquisas está circunscrita a serviços ou regiões específicas. Dentro dessa perspectiva, faz-se necessário dar maior visibilidade ao problema e, concomitantemente, fornecer os subsídios científicos necessários para orientar as ações de prevenção.

Considera-se, portanto, que a análise dos dados provenientes do Ministério da Saúde, pode contribuir de forma acentuada para aumentar a compreensão desse agravo. Dessa forma o presente estudo objetiva analisar a evolução das internações e taxa de mortalidade hospitalares de vítimas de queimaduras no Brasil, no período 2007-2016.

\section{MÉTODO}

Foi realizado um estudo observacional e retrospectivo, com dados secundários, obtidos do Sistema de Informações Hospitalares do Sistema Único de Saúde - SIH/ SUS do Brasil. Os dados coletados referiam-se ao período de 2007 a 2016.

O SIH/SUS tem a finalidade de registrar todos os atendimentos provenientes de internações hospitalares financiadas pelo Sistema Único de Saúde (SUS) e, por meio desse processamento, gerar relatórios para que os gestores possam encaminhar os pagamentos aos estabelecimentos de saúde. Seu documento-fonte é a Autorização de Internação Hospitalar (AlH).

Os dados foram extraídos do SIH-SUS, acessados via DATASUS, do Ministério da Saúde, nos meses de abril e maio de 2017. Foram consideradas as internações de vítimas de queimaduras, seguindo a $10^{\mathrm{a}}$ Revisão da Classificação Internacional de Doenças (CID- I0). Do capítulo XX da CID-10 - Causas externas de morbidade e de mortalidade - foram incluídas as internações decorrentes de exposição à corrente elétrica, à radiação e às temperaturas e pressões extremas do ambiente (W85-W99); exposição à fumaça, ao fogo e às chamas (X00-X09) e contato com uma fonte de calor ou substâncias quentes (XI0-XI9) e excluídos os dados de internação com sexo ou idade ignorados.

Foram analisadas variáveis relacionadas à morbimortalidade por queimaduras (internações, óbitos, mortalidade hospitalar, região geográfica e causa das queimaduras); à hospitalização (permanência hospitalar, valor médio da internação) e às vítimas de queimaduras (faixa etária e sexo).

Utilizou-se das ferramentas desenvolvidas pelo $\mathrm{Da}$ tasus - TabWin e TabNet - para a tabulação dos dados e programa computacional Stata versão 12.0 para análise estatística. As variáveis categóricas foram analisadas em forma de frequência simples e relativa e apresentadas em tabelas. Para avaliar as associações entre a ocorrência de óbito e as variáveis analisadas, foi realizada análise bivariada por meio do teste Qui-quadrado de Pearson $\left(X^{2}\right)$. Como medidas de força de associações, foram utilizados o risco relativo (RR) e intervalos de confiança de $95 \%$ (IC $95 \%)$. O nível de significância adotado foi de $5 \%(p \leq 0,05)$. 
Este estudo foi realizado exclusivamente com dados secundários e agregados, de acesso público, disponíveis on-line no site do Departamento de Informática do Sistema Único de Saúde (DATASUS). Dessa forma, o presente estudo se isenta do Termo de Consentimento Livre e Esclarecido.

\section{RESULTADOS}

Conforme apresentado na Figura I, foram registradas I.009.09| hospitalizações por queimaduras, com uma média anual de 100.909 internações, no período correspondente de 2007-2016. Observou-se uma tendência crescente das hospitalizações, com 6,0\% em 2007, avançando para 13,3\% em 2016. Em relação aos óbitos, foram registrados 20.907 casos, com taxa de mortalidade hospitalar média de 2,07\%, com destaque para o período de 2014 a 2016 , no qual ocorreram $37,2 \%$ dos óbitos.

Conforme disposto na Tabela I, pode-se verificar que o valor médio, em reais, gasto em cada hospitalização foi $\mathrm{R} \$ 1.105,27$, sendo observado um aumento deste valor no decorrer do período analisado. A permanência hospitalar apresentou uma média de 4,9 dias, com queda gradativa no período estudado. A menor taxa de mortalidade hospitalar foi verificada no ano de $2008(1,9)$, sendo a maior observada no ano de 201 I $(2,33)$.

Como pode ser observado na Tabela 2, houveram associações estatisticamente significativas entre o risco de óbito e o CID X00-X09 - exposição à fumaça, ao fogo

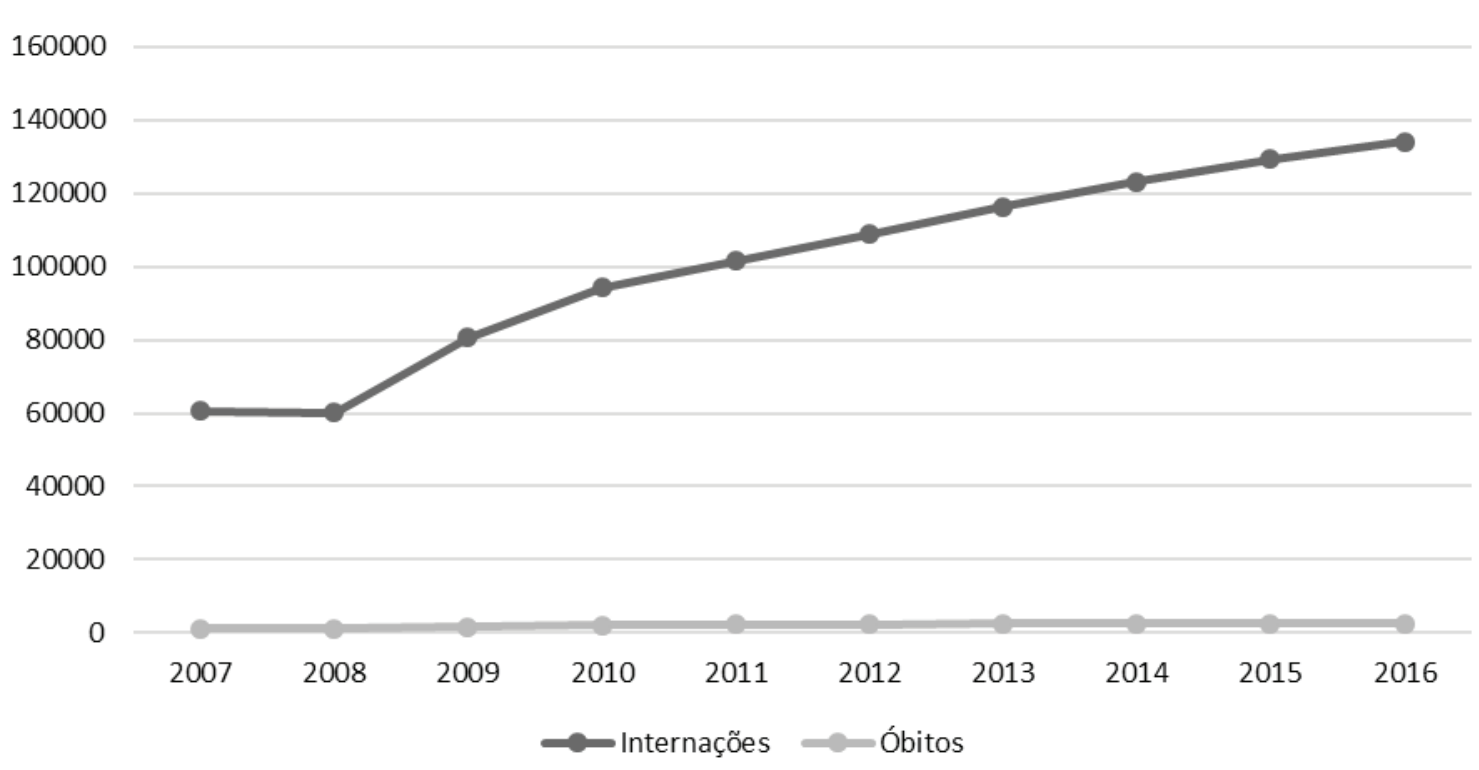

FIGURA 1 - Internações e óbitos por queimaduras no Brasil, 2007-2016.

Fonte: Ministério da Saúde - Sistema de Informações Hospitalares do SUS (SIH/SUS)

TABELA 1 - Permanência hospitalar e gastos com hospitalizações por queimaduras no Brasil no período de 2007 a 2016.

\begin{tabular}{c|c|c|c|c|c}
\hline Ano & Internações & Óbitos & Taxa Mortalidade & Valor $^{*}$ & Permanência† \\
\hline 2007 & 60.597 & 1.215 & 2,01 & 831,19 & 5,0 \\
\hline 2008 & 60.025 & 1.140 & 1,9 & 958,45 & 5,0 \\
\hline 2009 & 80.607 & 1.613 & 2 & $1.057,14$ & 4,8 \\
\hline 2010 & 94.387 & 2.027 & 2,15 & $1.095,86$ & 4,8 \\
\hline 2011 & 101.536 & 2.363 & 2,33 & $1.131,83$ & 5,1 \\
\hline 2012 & 108.957 & 2.300 & 2,11 & $1.118,99$ & 4,8 \\
\hline 2013 & 116.263 & 2.488 & 2,14 & $1.143,29$ & 4,9 \\
\hline 2014 & 123.130 & 2.521 & 2,05 & $1.165,94$ & 4,8 \\
\hline 2015 & 129.440 & 2.652 & 2,05 & $1.231,50$ & 4,8 \\
\hline 2016 & 134.149 & 2.588 & 1,93 & $1.318,60$ & 4,7 \\
\hline Total & 1.009 .091 & 20.907 & 2,07 & $1.105,27$ & 4,9 \\
\hline
\end{tabular}

* Média do valor gasto em reais

†Média da permanência em dias

Fonte: Ministério da Saúde - Sistema de Informações Hospitalares do SUS (SIH/SUS) 
e às chamas - $\left(p<0,00 I ; I C_{95 \%} 2,25 ; 2,4 I\right)$, sendo que os pacientes internados por esta causa apresentaram 2,3 vezes mais chance de óbito em relação às demais causas. No entanto, em relação aos internamentos predominaram as queimaduras do grupo de causas W85-W99 da CID-I0 (decorrentes da exposição a corrente elétrica, radiação, temperatura e pressão extremas), correspondendo a $84,1 \%$ dos casos, sendo essa mesma etiologia responsável pelo maior número de óbitos $(78,8 \%)$ na população brasileira no período analisado.

Quanto à distribuição espacial, observou-se associação estatisticamente significativa entre o risco de óbito

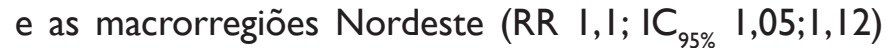

e Sudeste (RR I,4; IC ${ }_{95 \%}$ I,36; I,44). Constatou-se ainda uma distribuição heterogênea da morbimortalidade, com predomínio no sul e sudeste, responsáveis por $50,4 \%$ das internações e $62,4 \%$ dos óbitos. Em relação à faixa etária, verificou-se associação estatisticamente significativa entre a ocorrência de óbitos e idades superiores a 60 anos $(\mathrm{p}<0,00 \mathrm{I})$, sendo que a faixa etária de 80 anos e mais apresentou quase cinco vezes maiores chances de óbito em relação às demais ( $\left.R R \quad 4,9 ; 1 C_{95 \%} 4,74 ; 5,08\right)$. Predominaram internações em adultos ( 20 a 59 anos) com $58,2 \%$, seguidas por crianças e adolescentes (zero a 19 anos) com $27,2 \%$, já a faixa etária de 80 anos e mais foi responsável pelo maior número de óbitos (I4,5\%). Quanto

TABELA 2 - Características clinicas, geográficas e socioeconômicas das internações e óbitos no Brasil, no período de 2007 a 2016

\begin{tabular}{|c|c|c|c|c|c|c|c|c|}
\hline \multirow[t]{2}{*}{ Variáveis } & \multicolumn{2}{|c|}{ Internações } & \multicolumn{2}{|c|}{ Óbitos } & \multirow[t]{2}{*}{$\mathbf{X}^{2^{*}}$} & \multirow[t]{2}{*}{$p \dagger$} & \multirow[t]{2}{*}{ RR $\ddagger$} & \multirow[t]{2}{*}{ IC95§ } \\
\hline & $\mathbf{N}$ & $\%$ & $\mathbf{N}$ & $\%$ & & & & \\
\hline \multicolumn{9}{|l|}{ Causa } \\
\hline W85-W99 & 849.430 & 84,1 & 16.465 & 78,8 & 471,22 & \multirow{3}{*}{$<0,001 \|$} & 0,7 & 0,$72 ; 0,67$ \\
\hline X00-X09 & 80.466 & 8,0 & 3.510 & 16,8 & 22360,57 & & 2,3 & 2,$25 ; 2,41$ \\
\hline X10-X19 & 79.219 & 7,9 & 932 & 4,5 & 339,66 & & 0,5 & 0,$58 ; 0,51$ \\
\hline \multicolumn{9}{|l|}{ Macrorregião } \\
\hline Norte & 185.373 & 18,4 & 1.818 & 8,7 & 1332,57 & \multirow{5}{*}{$<0,001||$} & 0,4 & 0,$44 ; 0,40$ \\
\hline Nordeste & 193.505 & 19,2 & 4.266 & 20,4 & 20,79 & & 1,1 & 1,$05 ; 1,12$ \\
\hline Sudeste & 239.780 & 23,8 & 6.347 & 30,4 & 512,40 & & 1,4 & 1,$36 ; 1,44$ \\
\hline Sul & 267.969 & 26,6 & 6.692 & 32,0 & 325,49 & & 1,3 & 1,$27 ; 1,34$ \\
\hline Centro-Oeste & 122.464 & 12,1 & 1.783 & 8,5 & 259,92 & & 0,7 & 0,$71 ; 0,64$ \\
\hline \multicolumn{9}{|l|}{ Faixa-etária } \\
\hline Menor 1 ano & 9.077 & 0,9 & 180 & 0,9 & 0,44 & \multirow{12}{*}{$<0,001 \|$} & 1,0 & 1,$10 ; 0,82 \pi$ \\
\hline 1 a 4 anos & 54.980 & 5,4 & 312 & 1,5 & 654,17 & & 0,3 & 0,$29 ; 0,24$ \\
\hline 5 a 9 anos & 64.700 & 6,4 & 205 & 1,0 & 1056,55 & & 0,1 & 0,$16 ; 0,13$ \\
\hline 10 a 14 anos & 65.245 & 6,5 & 234 & 1,1 & 1016,08 & & 0,2 & 0,$18 ; 0,15$ \\
\hline 15 a 19 anos & 80.761 & 8,0 & 1.071 & 5,1 & 245,9 & & 0,6 & 0,$66 ; 0,58$ \\
\hline 20 a 29 anos & 190.655 & 18,9 & 3.031 & 14,4 & 281,11 & & 0,7 & 0,$75 ; 0,70$ \\
\hline 30 a 39 anos & 163.298 & 16,2 & 2.646 & 12,6 & 203,53 & & 0,7 & 0,$78 ; 0,72$ \\
\hline 40 a 49 anos & 131.056 & 13,0 & 2.768 & 13,2 & 0,68 & & 1,0 & 0,$98 ; 1,06 \pi$ \\
\hline 50 a 59 anos & 101.947 & 10,1 & 2.567 & 12,2 & 10,64 & & 0,9 & 0,$97 ; 0,90$ \\
\hline 60 a 69 anos & 67.253 & 6,7 & 2.378 & 11,3 & 747,37 & & 1,8 & 1,$71 ; 1,86$ \\
\hline 70 a 79 anos & 46.451 & 4,6 & 2.571 & 12,2 & 2848,11 & & 2,9 & 2,$78 ; 3,00$ \\
\hline 80 anos e mais & 33.692 & 3,3 & 3.045 & 14,5 & 8273,27 & & 4,9 & 4,$74 ; 5,08$ \\
\hline \multicolumn{9}{|l|}{ Sexo } \\
\hline Masculino & 695.236 & 68,9 & 14.258 & 68,2 & 4,86 & \multirow{2}{*}{$0,028 \|$} & 1,0 & 1,$0 ; 0,94$ ๆ \\
\hline Feminino & 313.879 & 31,1 & 6.649 & 31,8 & 4,78 & & 1,0 & 1,$0 ; 1,06$ \\
\hline
\end{tabular}

* Teste Qui-quadrado de Pearson

† Nível de significância adotado foi de $5 \%(p \leq 0,05)$

$\ddagger$ Risco Relativo (RR)

$\S$ Intervalo de confiança de 95\% (IC 95\%)

||Associação estatística significativa no nível de confiança de 95\%

I Associação não significativa no IC95\%

Fonte: Ministério da Saúde - Sistema de Informações Hospitalares do SUS (SIH/SUS) 
ao sexo, o masculino predominou tanto em internações $(68,9 \%)$ quanto em mortalidade $(68,2 \%)$.

\section{DISCUSSÃO}

Os achados do presente estudo apontam para um aumento das internações por queimaduras no Brasil entre os anos de 2007 e 2016 . Em contrapartida, a taxa de mortalidade hospitalar sofreu pouca variação, declinando a partir de 2014. Tais achados podem relacionar-se às melhorias no atendimento às urgências e emergências no Brasil, com a implantação da Rede de Atenção às Urgências a partir do ano de $201 \mathrm{l}$.

Nesse contexto, pode-se destacar a ampliação das equipes e, consequentemente do acesso à população ao SAMU nos últimos anos. A atuação eficaz deste serviço, contribui para a redução no número de óbitos, das sequelas resultantes da demora non atendimento de urgência e tempo de internação hospitalar ${ }^{(6)}$. Além disso, avanços obtidos no atendimento inicial ao queimado nos últimos anos, como a reposição volêmica adequada, contribuíram significativamente para a melhora no prognóstico das vítimas ${ }^{(7)}$.

No entanto, apesar de tais avanços, as queimaduras estão entre as lesões não letais mais onerosas que demandam preocupações quanto à assistência às vítimas, devido aos altos custos associados ao tratamento e longos períodos de permanência hospitalar ${ }^{(8)}$. Em estudo realizado na Austrália foi encontrado um valor médio superior a 3.600 dólares por dia de internação ${ }^{(9)}$. Já em outro estudo conduzido em um centro de tratamento de queimados da Holanda, encontrou-se valor médio de 26.540 euros no tratamento integral de tais pacientes ${ }^{(10)}$. Os valores observados nesses países são bem maiores aos gastos no Brasil, indicando maior investimento no cuidado ao paciente com queimaduras em países desenvolvidos.

O tempo de permanência hospitalar das vítimas de queimaduras deste estudo foi inferior comparado a estudos realizados na Colômbia ${ }^{(7)}$, China ${ }^{(11)}$ e também no Brasil $^{(12)}$, que variou de 12 a 25 dias, sendo observadas variações no tempo de permanência hospitalar relacionadas a fatores como sexo e faixa etária. No entanto, cabe ressaltar, que estudos supracitados foram realizados em serviços especializados no tratamento de pacientes queimados, já que o presente estudo engloba hospitalizações por queimaduras ocorridas no Brasil e inseridas no $\mathrm{SIH} /$ SUS, independente da complexidade da instituição. Outro estudo realizado em Portugal, encontrou uma média de nove dias de hospitalização em pacientes queimados atendidos em hospitais excluindo centros especializados (8), ainda assim superior encontrado neste estudo.

No que se refere à etiologia das queimaduras, predominaram as internações e óbitos decorrentes da exposição à corrente elétrica, à radiação e às temperaturas e pressões extremas do ambiente. A utilização do grupo de causas da CID-I0 dificultou a comparação com outros estudos que relacionam as queimaduras a circunstâncias e agentes causais específicos. Outro estudo brasileiro, referente a atendimentos de urgência, mostrou que o contato com substâncias quentes foi a principal causa da queimadura, seguido pela exposição ao fogo e às chamas ${ }^{(1)}$.

Nesse contexto, merecem ser destacados os eventos relacionados a incêndios no país, pois muitas vezes o número de vítimas ultrapassa a quantidade de recursos locais disponíveis para o atendimento das mesmas. Cabe ressaltar o incidente com múltiplas vítimas ocorrido em 2013 em um município da região sul do Brasil, classificado como a quinta maior tragédia e o segundo maior incidente de incêndio no Brasil nos últimos 50 anos. Este evento resultou na morte de 242 pessoas, em sua maioria estudantes universitários, devido a asfixia e intoxicação por monóxido de carbono e cianeto(13).

A discrepância regional em relação às internações $e$ mortalidade por queimaduras no Brasil, com destaque para as regiões sul e sudeste, pode estar relacionada ao maior contingente populacional dessas regiões. No entanto, em relação a oferta de serviços especializados, embora a região sul do Brasil possua a liderança no ranking das internações e mortalidade, conta com apenas sete UTQs, enquanto o estado de São Paulo concentra 16 dessas unidades ${ }^{(4)}$.

Ademais, a região do nordeste brasileiro que ocupou o terceiro lugar na morbimortalidade por queimaduras, conta com um cenário lastimável em que óbitos ocasionados pelo fogo, de maneira intencional, são uma ameaça constante, e por vezes banalizada, no cotidiano das mulheres em situação de vulnerabilidade social. Não obstante, a discussão acerca do tratamento das lesões por queimaduras é por vezes negligenciada nas políticas públicas e debates acadêmicos ${ }^{(14)}$.

A idade das vítimas de queimaduras parece variar nos estudos brasileiros, uma vez que a maioria é realizada com populações locais e em serviços específicos ${ }^{(15)}$. $O$ maior número de internações na população economicamente ativa observado neste estudo é relevante visto que em países de baixa e média rendas, como o Brasil, um percentual considerável dos acidentes não fatais são incapacitantes devido às sequelas produzidas ${ }^{(16)}$. Destarte, é relevante o impacto nos anos de vida perdidos ajustados por incapacidades - DALYs- decorrentes desse tipo de injúria. Segundo dados da Organização Mundial de Saúde, as queimaduras são responsáveis por aproximadamente 18 milhões de DAYLs, com mais de $90 \%$ concentrados em países de baixa e média rendas ${ }^{(17)}$.

As crianças constituem também um grupo importante para ocorrência desses agravos. Em estudo brasileiro realizado em 86 serviços de saúde sentinelas no atendimento 
das urgências e emergências, observou-se que as queimaduras ocuparam $\circ 4^{\circ}$ lugar entre os atendimentos por causas externas em crianças de zero a nove anos, sendo mais frequentes entre aquelas com até um ano de idade ${ }^{(18)}$.

Em relação à mortalidade destacaram-se os idosos com 80 anos e mais. Um estudo chinês, realizado em um período de 10 anos, com vítimas de queimaduras, encontrou maiores taxas de mortalidade entre os idosos, em especial na faixa etária de 60-69 anos ${ }^{(1)}$. Nesse contexto, as lesões por queimaduras em idosos, em comparação com indivíduos mais jovens, ocasionam maior comprometimento físico, redução da qualidade de vida, perda da independência e aumento da mortalidade.Além disso, os idosos possuem chances mais elevadas de óbito no primeiro e segundo ano após a queimadura. Uma coorte australiana encontrou $29 \%$ de mortalidade a longo prazo atribuída à queimadura ${ }^{(19)}$.

De modo geral, as queimaduras obtiveram uma taxa de mortalidade intra-hospitalar média de $2,07 \%$ no Brasil no período de estudo. Este valor é inferior à taxa de mortalidade de Portugal $(4,4)^{(8)}$.

Considerando o sexo das vítimas, a morbimortalidade por queimaduras foi predominante entre os homens, corroborando com a literatura nacional, latino-americana, asiática e europeia ${ }^{(5,7,11,13)}$. Tal fato pode ser justificado, principalmente, pelo comportamento de risco masculino, pelo perfil das atividades laborais desenvolvida pelos homens e, no caso das crianças, pela maior liberdade concedida pelos pais e maior impulsividade do gênero masculino ${ }^{(7-8,11)}$.

Em contrapartida, a literatura internacional tem demonstrado uma forte associação entre vulnerabilidade social e de gênero com maiores taxas de morbimortalidade por queimaduras. Nesse contexto, a ocorrência de queimaduras associa-se com baixos níveis de renda em todos os países e, mesmo em países com média e altas rendas as queimaduras relacionam-se desproporcionalmente em minorias raciais e étnicas em que a condição socioeconômica aliada a fatores culturais e educacionais representam maior susceptibilidade à ocorrência de queimaduras. Entre os grupos em situações de vulnerabilidade é marcante a maior ocorrência de queimaduras entre as mulheres ${ }^{(20)}$. Tal fato, no entanto, não pôde ser investigado no presente estudo, uma vez que tais variáveis não se estão disponíveis no SIH-SUS.

A realização de estudos sobre morbimortalidade com dados provenientes do SIH/SUS é importante para conhecer a magnitude do problema investigado. $O$ levantamento de dados referentes a morbimortalidade hospitalar das queimaduras ocorridos no país, pode contribuir para a construção de indicadores que permitem uma aproximação da situação de saúde da população e do risco de morte. No entanto, algumas limitações inerentes à utilização do SIHSUS merecem ser destacadas, como o sub-registro e subnotificação de casos e o preenchimento inadequado ou incompleto de dados.

O estudo possui ainda limitações no sentido de que variáveis importantes para a análise da casuística das queimaduras, tais como percentual da superfície corporal queimada, grau e topografia das lesões, e local da ocorrência não puderam ser identificados por meio da base de dados utilizada. Esse estudo não objetivou realizar uma análise de tendência linear, portando não se pode identificar se a tendência de morbimortalidade é significativa de forma crescente ou estacionária. Em contrapartida, o presente estudo forneceu importantes contribuições sobre como se apresentam internações e óbitos por queimaduras em um período de 10 anos no Brasil, em relação aos anos de estudo, permanência e custos hospitalares, divisão regional, causa das queimaduras, faixa-etária e sexo das vítimas.

\section{CONCLUSÕES}

Ressalta-se a importância de destacar que no período investigado houve aumento das internações e óbitos por queimaduras no Brasil. A taxa de mortalidade hospitalar apresentou pouca variação, com discreto declínio a partir de 2014. A morbimortalidade hospitalar teve como causa principal a exposição a corrente elétrica, radiação, temperatura e pressão extremas e foi predominante nas regiões sul e sudeste do país. Predominaram internados adultos do sexo masculino e os óbitos destacaram-se entre idosos com 80 anos e mais. $O$ tempo médio das internações foi de quase cinco dias, implicando gastos expressivos.

Os resultados apresentados mostram múltiplas dimensões das internações e óbitos por queimaduras na população brasileira e reforçam a necessidade premente de outros estudos para fomentar a formulação de políticas públicas de prevenção desses agravos, principalmente na população economicamente ativa masculina.As queimaduras, sejam acidentais ou relacionadas à violência, ocasionam custos elevados para o sistema de saúde, perda da produtividade decorrente das sequelas e mortes prematuras, além do impacto familiar e social. Destarte, é necessário incluir esse assunto nos debates governamentais e acadêmicos, promovendo estratégias articuladas com os setores da educação, saúde e segurança, com o envolvimento da sociedade civil, com o intuito de promover propostas robustas de prevenção e tratamento das queimaduras em todo território nacional.

Considerando que na literatura nacional são escassos os estudos que abordam a relação entre a ocorrência de queimaduras e questões de vulnerabilidade e gênero, configurando o problema como uma lacuna na literatura científica nacional. Dessa forma, sugere-se a realização de estudos envolvendo a perspectiva de gênero e vulnerabilidade buscando clarear a influência desses fatores na morbimortalidade por queimaduras no Brasil. 


\section{REFERÊNCIAS}

I. Gawryszewski VP, Bernal RTI, Silva NN, Morais Neto OL, Silva MMA, Mascarenhas MDM et al. Public hospital emergency department visits due to burns in Brazil, 2009. Cad. Saúde Pública [Internet]. 2012 [acesso em 10 abr 2016]; 28(4): 629-40. Disponível em: http://www.scielo.br/pdf/csp/ v28n4/03.pdf.

2. Wallace HJ, O'Neill TB, Wood FM, Edgar DW, Rea SM. Determinants of burn first aid knowledge: Cross-sectional study. Burns [Internet]. 2013 [acesso em 10 abr 2016]; 39(6): I 162-9. Disponível em: https://pubmed.ncbi.nlm.nih. gov/23590966/.

3. World Health Organization (WHO). Fact sheet on burns [Internet] 2018. [access Jan 13, 2014].Available from: http:// www.who.int/mediacentre/factsheets/fs365/en/.

4. Silva JAC, Lima AVM, Borborema CLP, Cunha LM, Martins MM, Pantoja MS. Perfil dos pacientes queimados atendidos em um centro de referência na região metropolitana de Belém do Pará. Rev Bras Queimaduras [Internet]. 2016 [acesso em 10 abr 2016]; 15(3):I53-7. Disponível em: http://www.rbqueimaduras.com.br/details/3। I/pt -BR/perfil-dos-pacientes-queimados-atendidos-em-umcentro-de-referencia-na-regiao-metropolitana-de-belem-do-para.

5. Gervasi LC, Tibola J, Schneider IJC. Tendência de morbidade hospitalar por queimaduras em Santa Catarina. Rev Bras Queimaduras [Internet]. 2014 [acesso em 10 abr 2016]; I3(I):3 |-7. Disponível em: http://www.rbqueimaduras.com. br/details//83/pt-BR/tendencia-de-morbidade-hospitalar -por-queimaduras-em-santa-catarina.

6. Almeida PMV, Dell'Acqua MCQ, Cyrino CMS, Juliani CMCM, Palhares VC, Pavelqueires S. Analysis of services provided by SAMU 192: Mobile component of the urgency and emergency care network. Esc. Anna Nery [Internet]. 2016 [accesso em 10 abr 2016]; 20(2): 289-95. Disponível em: http://www.scielo.br/pdf/ean/v20n2/en_|4|4-8|45-ean-20-02-0289.pdf.

7. Ramírez-Blanco CE, Ramírez-Rivero CE, Días-Martiínez LA. Agent and survival in burn patients on the referral center of northeastern Colombia. Cir. plást. iberolatinoam [Internet]. 2017 [acesso em I 2 jul 20I6]; 43(I):59-67. Disponível em: http://scielo.isciii.es/pdf/cpil/v43n I/quemados I.pdf.

8. Santos JV, Oliveira A, Costa-Pereira A, Amarante J, Freitas A. Burden of burns in Portugal, 2000-2013: a clinical and economic analysis of 26,447 hospitalisations. Burns [Internet]. 2016 [access Jul 12, 2016]; 42(4):891-900. Available from: http://www.burnsjournal.com/article/S03054I79(16)00039-5/fulltext.

9. Ahn CS, Maitz PKM. The true cost of burn. Burns [Internet]. 2012 [Access Jul 12, 2016]; 38(7): 967-74. Available from: http://www.burnsjournal.com/article/S0305-4I 79(I2)00I74-X/fulltext.

10. Hop MJ, Wijnen BFM, Nieuwenhuis MK, Dokter J, Mi- ddelkoop E, Polinder S, Van Baar M. Economic burden of burn injuries in the Netherlands: a 3 months follow-up study. Injury [Internet]. 2016 [access ]ul 12, 2016]; 47(I): 20321. Available from: http://www.injuryjournal.com/article/ S0020-I383(I5)00547-I/pdf.

I I. Fan X, Ma B, Zeng D, Fang X, Li H, Xiao S,Wang G,Tang H, Xia Z. Burns in a major burns center in East China from 2005 to 2014: Incidence and outcome. Burns [Internet]. 2017 [access Jul 12, 2016]; 43(7): I586-95. Available from: http:// www.burnsjournal.com/article/S0305-4I 79(I 7)30074-8/ fulltext.

12. Queiroz LF, Anami EH, Zampar EF, Tanita MT, Cardoso LT, Grion CM. Epidemiology and outcome analysis of burn patients admitted to an intensive care unit in a university hospital. Burns [Internet]. 2016 [access Jul I2, 2016];42(3):65562. Available from: http://www.burnsjournal.com/article/ S0305-4I79(I5)002 I2-0/fulltext.

13. Gragnani A, Oliveira AF, Boro D, Pham TN, Ferreira LM. Response and legislative changes after the Kiss nightclub tragedy in Santa Maria/RS/Brazil: learning from a large-scale burn disaster. Burns [Internet]. 2017 [access Jul I2, 2016]; 43(2):343-9. Available from: http://www.burnsjournal.com/ article/S0305-4I79(I6)30288-I/fulltext.

14. Arruda CN, Braide ASG, Nations M. "Raw and charred flesh": the experience of burned women in Northeast Brazil. Cad. Saúde Pública [Internet]. 2014 [access Aug 10, 2016]; 30(I0): 2057-67.Avaiable from: http://www.scielo.br/ pdf/csp/v30n I0/0 I02-3 I IX-csp-30-10-2057.pdf.

15. Lima KRB, Oliveira SP, Rodriguez GCB, Nascimento RA, Nunes LM, Aiquoc KM, et al. Characterization of publications on burns in Brazil and changes resulting from trauma. International Archives of Medicine [Internet]. 2017 [access Aug 10, 2016]; 9(sn): I-8. Available from: https://imed.pub/ ojs/index.php/iam/article/view/2045/2000.

16. Meschial WC, Oliveira MLF. Initial care for burned patients in academic nursing education. Rev Rene [Internet]. 2017 [access Aug 10, 2016]; 17(2):212-9. Avaiable from: http:// www.revistarene.ufc.br/revista/index.php/revista/article/ viewFile/2606/pdf.

17. Rybarczyk MM, Schafer JM, Elm CM, Sarvepalli S, Vaswani PA, Balhara KS, et al. Prevention of burn injuries in lowand middle-income countries: a systematic review. Burns [Internet]. 2016 [access Aug 10, 2016]; 42(6): I I83-92. Available from: http://www.burnsjournal.com/article/S03054I79(16)30077-8/fulltext.

18. Malta DC, Mascarenhas MDM, Silva MMA, Carvalho MGO, Barufaldi LA, Avanci JQ, et al. The occurrence of external causes in childhood in emergency care: epidemiological aspects, Brazil, 20I4. Ciênc. saúde coletiva [Internet]. 2016 [access Aug 10, 2016]; 2I(I2): 3729-44. Avaiable from: http://www.scielo.br/pdf/csc/v2 In I2/en_I4I3-8I23csc-21-12-3729.pdf.

19. Duke JM, Boyd JH, Rea S, Randall SM, Wood FM. Long-term 
mortality among older adults with burn injury: a population -based study in Australia. Bull World Health Organ [Internet]. 2015 [access Aug 10,2016];93(6):400-6.Available from: http://www.who.int/bulletin/volumes/93/6/I4-|49|46/en/.

20. Dissanaike S, Ha D, Mitchell D, Larumbe E. Socioeconomic status, gender, and burn injury: a retrospective review.Am J
Surg [Internet]. 2017 [access Aug 10, 20I6]; 214(4):677-8I. Available from: http://www.americanjournalofsurgery.com/ article/S0002-96I0(I7)30I I4-9/fulltext.

Recebido: 2020-07-23

Aceito: $2020-08-28$ 